\title{
Rescuing p53 from mdm 2 by a pre-structured motif in intrinsically unfolded SUMO specific protease 4
}

\author{
Do-Hyoung Kim, Chewook Lee, Bom Kim, Si-Hyung Lee \& Kyou-Hoon Han * \\ Korea Research Institute of Bioscience and Biotechnology, Daejeon 34141, Korea
}

\begin{abstract}
Many intrinsically unstructured/unfolded proteins (IUPs) contain transient local secondary structures even though they are "unstructured" in a tertiary sense. These local secondary structures are named "pre-structured motifs (PreSMos)" and in fact are the specificity determinants for IUP-target binding, i.e., the active sites in IUPs. Using high-resolution NMR we have delineated a PreSMo active site in the intrinsically unfolded mid-domain (residues 201-300) of SUMO-specific protease 4 (SUSP4). This 29-residue motif which we termed a p53 rescue motif can protect p53 from $\mathrm{mdm} 2$ quenching by binding to the p53-helix binding pocket in mdm2(3-109). Our work demonstrates that the PreSMo approach is quite effective in providing a structural rationale for interactions of p53-mdm2SUSP4 and opens a novel avenue for designing mdm2inhibiting anticancer compounds. [BMB Reports: Perspective 2017; 50(10): 485-486]
\end{abstract}

With its tumor-suppressive activity, p53 has gotten a cognomen, "the guardian of genome", and perhaps is the most well-known protein in cancer-related fields. Peculiarly, the transactivation domain (TAD) of p53 is "unstructured", i.e., does not form a globular structure, which is rather unusual for a 73-residue long domain. To be more precise, p53 TAD should be described as being intrinsically unfolded, not simply "unstructured", since it contains transient local secondary structures although its overall topology is ill-defined being devoid of a spatially unique three-dimensional structure.

*Corresponding author. E-mail: khhan600@kribb.re.kr

https://doi.org/10.5483/BMBRep.2017.50.10.131

Received 13 July 2017

Keywords: IUP, Mdm2, p53, PreSMo, SUSP4

Abbreviations: IUP, intrinsically unfolded protein; Mdm2, mouse double minute 2; PreSMo, pre-structured motif; SUSP4, sumo-specific protease 4

Perspective to: Kim et al., The Mechanism of p53 Rescue by SUSP4. Angew. Chem. Int. Ed. 2017, Jan 24;56(5):1278-1282. doi: 10.1002/ anie.201607819. Epub 2016 Dec 21.
When one considers only a structural aspect, p53 TAD resembles a pre-molten globule which is inactive in a functional sense.

A rather astonishing fact is that the intrinsically unfolded p53 TAD and in fact many other TFs or TADs resembling a pre-molten globule exist in such a structural state under non-denaturing conditions $(\sim \mathrm{pH} 7, \mathrm{PBS}$, room temperature etc), and hence are functional. These unorthodox proteins or long protein regions (the word "region" is used instead of "domain" since the latter tends to insinuate presence of a globular structure) that are functional without a 3-D structure are now termed intrinsically unfolded/unstructured proteins (IUPs) or intrinsically disordered proteins (IDPs), and defy the golden structure-function paradigm in protein science. The transient local secondary structures, recently coined as pre-structured motifs (acronymed as a "PreSMo"), are in fact observed in dozens of IUPs/IDPs including several viral or neurodegenerative IUPs/IDPs and are the key determinants within the flexible IUPs/IDPs for target-binding. Presence of PreSMos in IUPs/IDPs elegantly argues that the target-binding of IUPS/IDPs is mediated by specific motifs, not by the unfolded nature per se of IUPs/IDPs. Table 1 provides a few examples of PreSMos.

SUSP4 is a mouse homolog of SENP2 that promotes mdm2 self-ubiquitination by removing SUMO-1 from $\mathrm{mdm} 2$, resulting in restoration of the transcriptional activity of p53 from mdm2 attack. An earlier study showed that the mid-domain (residues 201-300) of SUSP4 binds to the N-terminal globular domain of $\mathrm{mdm} 2$ (residues 1-150) that contains the p53 binding interface. Protein disorder prediction algorithms reveal that the N-terminal 300 residues of SUSP4 are intrinsically unfolded including the (201-300) region that binds mdm2. Using heteronuclear multidimensional NMR and other biophysical techniques we have determined the mechanism of p53 rescue by SUSP4 the intrinsically disordered 100-residue mid-domain of SUSP4 contains a pre-structured "p53 rescue motif" (Diagram 1).

\section{ACKNOWLEDGEMENTS}

This work was supported by an NST grant (NTC2251422) and a grant from the R\&D program for cancer control, Ministry of Health and Welfare, Republic of Korea (1320190) to K.H. 
Rescuing $\mathrm{p} 53$ from mdm2 by intrinsically unfolded SUMO protease 4 Do-Hyoung Kim, et al.

Table 1. Examples of pre-structured motifs (PreSMos) observed in some well-known IUPs/IDPs

\begin{tabular}{|c|c|c|c|c|c|}
\hline Name & $\begin{array}{l}\text { Number of } \\
\text { residues }^{\mathrm{a}}\end{array}$ & PreSMo-forming residues ${ }^{b}$ & $\begin{array}{l}\text { Population }{ }^{\mathrm{c}} \\
(\%)\end{array}$ & Binding partners & Remarks \\
\hline p53 TAD & $73(1-73)$ & $\begin{array}{l}\text { 18-26: H } \\
\text { 40-44/48-53: T }\end{array}$ & $\begin{array}{c}20 \\
5-15\end{array}$ & Mdm2, RPA, TFII & J. Biol. Chem. (2000) 275, 29426 \\
\hline VP16 TAD & $79(412-490)$ & $\begin{array}{l}\text { 424-433: H } \\
\text { 442-447/465-483: } \mathbf{H}\end{array}$ & $\begin{array}{r}60 \\
\sim 20\end{array}$ & $\mathrm{hTAF}_{\|} 31, \mathrm{PC} 4$ & $\begin{array}{l}\text { Biochemistry (2005) 44, 827; } \\
\text { BMB Rep. (2009) 42, } 411\end{array}$ \\
\hline CFTR & $185(654-838)$ & $\begin{array}{l}\text { 654-668/759-764: H } \\
\text { 766-776/801-817: H } \\
744-753: \beta\end{array}$ & $\begin{array}{l}>5 \\
>5 \\
>5\end{array}$ & NT-binding domain 1 & Nat. Struct. Mol. Biol. (2007) 14, 738 \\
\hline HBV preS1 & $119(1-119)$ & $\begin{array}{l}32-36,41-45,11-18,22-25 \\
37-40,46-50\end{array}$ & $\begin{array}{l}\sim 10 \\
\sim 10\end{array}$ & Hepatocyte receptor & Protein Sci. (2007) 16, 2108 \\
\hline Securin & $202(1-202)$ & $\begin{array}{l}\text { 150-159: } \mathbf{H} \\
113-127: \boldsymbol{\beta} \\
174-178:-\end{array}$ & $\begin{array}{l}45 \\
15 \\
20\end{array}$ & - & J. Am. Chem. Soc. (2008) 130, 16873 \\
\hline MSP2 & $237(1-237)$ & $\begin{array}{l}\text { 14-21: } \mathbf{H} \\
\text { 140-150: } \mathbf{H} \\
\text { 197-211 }\end{array}$ & $\begin{array}{l}35 \\
35 \\
20\end{array}$ & Lipid & J. Mol. Biol. (2008) 379, 105 \\
\hline Tau & $441(1-441)$ & $\begin{array}{l}\text { 114-123/428-437: } \mathbf{H} \\
\text { 274-284/305-315/336-345: } \boldsymbol{\beta} \\
\text { 175-184/216-223/232-239: PPII }\end{array}$ & & Microtuble & PLoS Biol. (2009) 7, e1000034 \\
\hline SUSP4 & $100(201-300)$ & 263-291: H & $\sim 30$ & Mdm2 & Current work \\
\hline
\end{tabular}

${ }^{\mathrm{a}}$ The numbers indicate the total number of amino acid residues in IUPS/IDPs and those in parenthesis are the sequential residue numbers. ${ }^{\mathrm{b}}$ The symbols $\mathbf{H}, \mathbf{T}, \boldsymbol{\beta}$ and PPII denote helix, turn, $\beta$-strand, and polyproline II helix, respectively. No symbol is given when the nature of the secondary structure is not described in the original report. 'The population is obtained from NMR chemical shifts using the SSP (secondary structure propensity) calculation algorithm of Marsh et al., (2006).

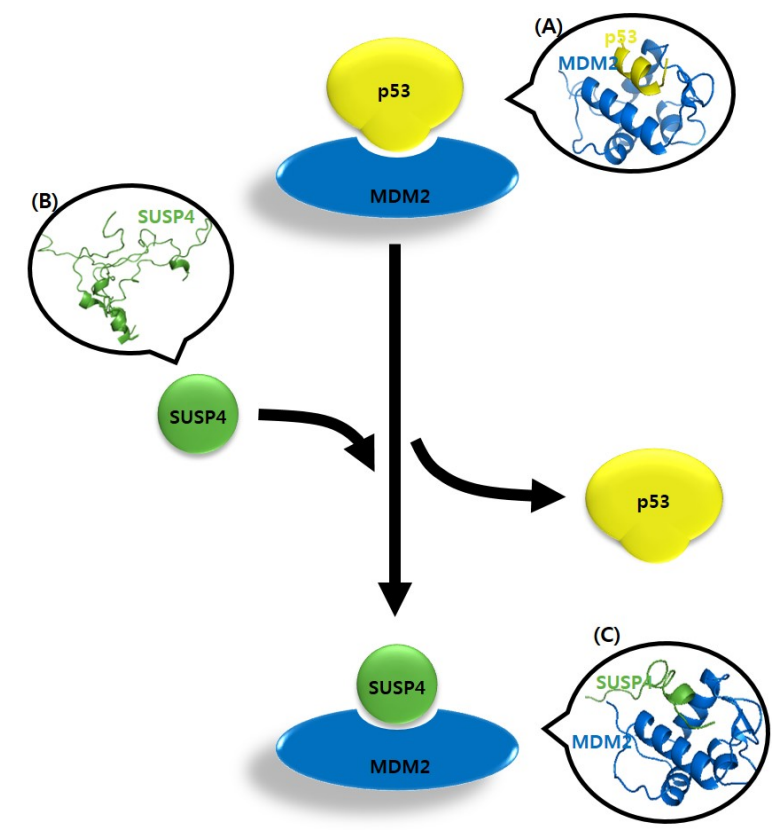

Diagram 1. A scheme describing p53 rescue by the p53 rescue motif found in the intrinsically unfolded SUSP4. (A) The helix PreSMo in p53 TAD (yellow) is bound by mdm2 (blue). This PreSMo is depicted as a bulge at the bottom of p53 in the top cartoon. (B) An ensemble representation of the disordered SUSP4 29-mer PreSMo (termed "p53 rescue motif") populated with a helix (green). (C) The p53 rescue motif of SUSP4 (green) has replaced the p53 helix and is bound to mdm2. 